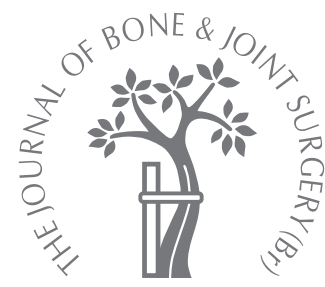

- CASE REPORT

\title{
Costo-osteochondral graft for post-traumatic osteonecrosis of the radial head in an adolescent boy
}

\author{
S. Iwai, \\ K. Sato, \\ T. Nakamura, \\ M. Okazaki, \\ Y. Itoh, \\ Y. Toyama, \\ H. Ikegami
}

From Department of

Orthopaedic Surgery,

School of Medicine,

Keio University,

Tokyo, Japan

\footnotetext{
S. Iwai, MD, Orthopaedic

Surgeon

K. Sato, MD, PhD,

Orthopaedic Surgeon

Assistant Professor

- T. Nakamura, MD, PhD,

Orthopaedic Surgeon,

Assistant Professor

M. Okazaki, MD, Orthopaedic

Surgeon

Y. Toyama, $\mathrm{MD}, \mathrm{PhD}$

Orthopaedic Surgeon,

Professor

H. Ikegami, MD, PhD,

Orthopaedic Surgeon

Assistant Professor

Department of Orthopaedic

Surgery

School of Medicine, Keio

University, 35 Shinanomachi,

Shinjuku-ku, Tokyo 160-8582,

Japan.

Y. Itoh, MD, PhD, Orthopaedic

Surgeon

Keiyu Orthopaedic Hospital,

1741 Hanetsukucho,

Tatebayashi, Gunma 374-0011,

Japan.

Correspondence should be sent to Professor K. Sato; e-mail:

kazuki@sc.itc.keio.ac.jp

(C)2011 British Editorial Society of Bone and Joint Surgery doi:10.1302/0301-620X.93B1. $25130 \$ 2.00$

J Bone Joint Surg [Br] 2011;93-B:111-14.

Received 8June 2010; Accepted after revision 23 September 2010
}

\author{
We present a case of post-traumatic osteonecrosis of the radial head in a 13-year-old boy \\ which was treated with costo-osteochondral grafts. A satisfactory outcome was seen at a \\ follow-up of two years and ten months. \\ Although costo-osteochondral grafting has been used in the treatment of defects in \\ articular cartilage, especially in the hand and the elbow, the extension of the technique to \\ manage post-traumatic osteonecrosis of the radial head in a child has not previously been \\ reported in the English language literature. Complete relief of pain was obtained and an \\ improvement in the range of movement was observed. The long-term results remain \\ uncertain.
}

A 13-year-old boy presented with pain and a severely restricted range of movement at his left elbow. He had suffered a fracture dislocation of the elbow, a fracture of the radial neck, and a distal radial fracture in a fall five months earlier. Initial open reduction and internal fixation using Kirschner (K)-wires was performed at another hospital. Mobilisation had started three weeks post-operatively and the K-wires were removed six weeks postoperatively. However, he developed increasing pain and limitation of movement. There was a fixed-flexion deformity of $40^{\circ}$ and flexion to $90^{\circ}$. There was no pronation of the forearm and $30^{\circ}$ of supination. There was marked valgus instability and crepitus. Radiographs showed destructive changes of the radial head which now comprised a collection of bone fragments. The radius had shortened and undergone proximal migration (Fig. 1). On MRI, fragments of the radial head demonstrated low signal intensity on the T1-weighted image and high signal intensity on the T2-weighted image, which was suggestive of necrosis (Fig. 2).

Reconstruction of the radial head was performed at nine months post-operatively, using a costo-osteochondral graft. Exposure of the radiohumeral joint was obtained through a lateral approach. The fragmented radial head was enclosed in scar tissue with no evidence of any remaining viable bone or cartilage.

The proximal migration of the shaft of the radius was associated with damage to the articular surface of the capitellum. After removal of the necrotic fragments and scar tissue, two pieces of osteochondral graft were harvested from the costo-osteochondral junctions of the left 4th and 5 th ribs through a transverse sub-mammary incision, approximately $5 \mathrm{~cm}$ in length. Care was taken to avoid perforating the pleura. The whole costo-osteochondral junctions were harvested en bloc. The osteochondral pieces were joined with low profile screws and shaped to the form of a radial head (Fig. 3). The cartilage in the graft was trimmed using a scalpel to match the capitellum with adequate contour. The proximal end of the radius was step-cut to provide a graft-bed using a saw and chisel. The osseous part of the graft was shaped to match the step-cut in the radius. After confirming the congruity of the joint, the graft was secured with mini screws. An external fixation device which allowed movement of the elbow was applied.

Post-operative radiographs showed a radiolucent area of the new radial head, which appeared quite large because of the thickness of the chondral portion of the graft (Fig. 4). After surgery, the elbow was immobilised with a plaster cast in $90^{\circ}$ of elbow flexion and neutral position of forearm rotation for two weeks, after which the cast was removed and mobilisation commenced with the protection of the articulated external fixator. The external fixation device was removed two months postoperatively.

Seven months post-operatively a screw was removed because of loosening. At this operation the reconstructed radial head seemed to 


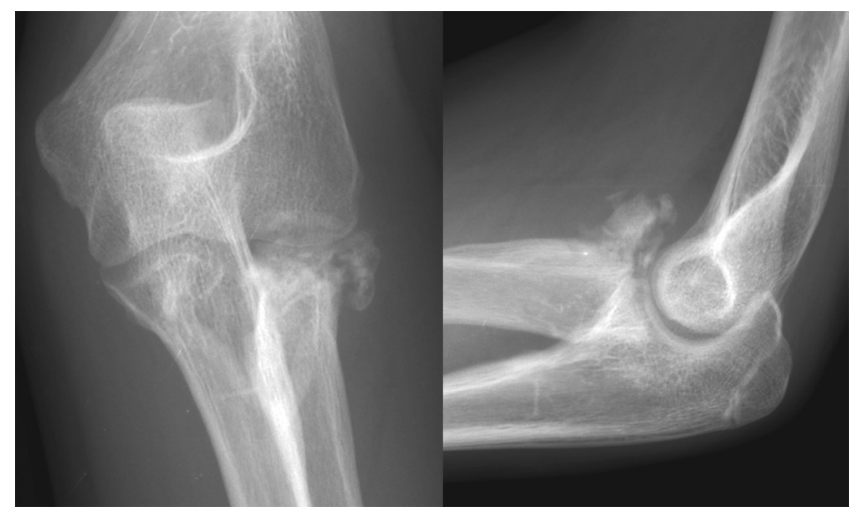

Fig. 1

Anteroposterior lateral radiographs of the left elbow at presentation five months after the initial surgery, showing the displaced fragments of the radial head. The radius has migrated proximally. There is some erosion of the capitellum.

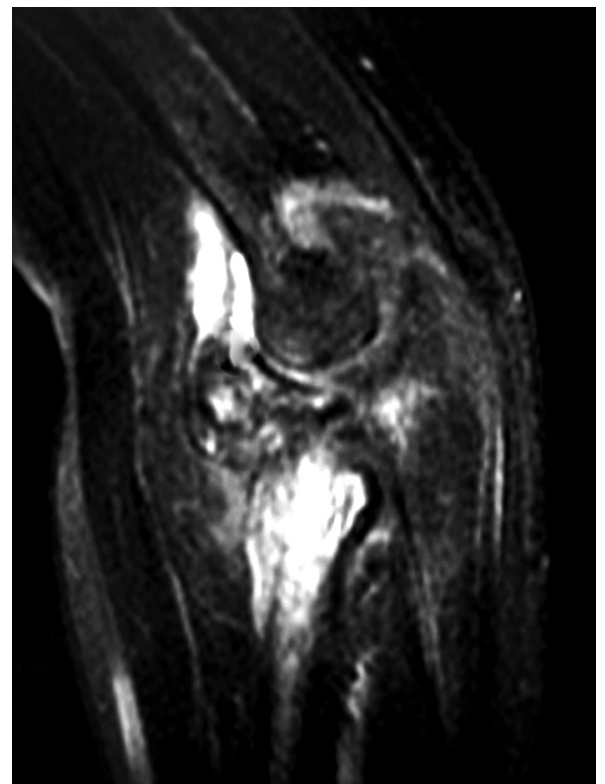

Fig. 2

MRI demonstrating necrotic change of the radial head with high signal intensity on T2-weighted image.

have retained its shape. The articular surface was white and smooth, resembling normal articular cartilage (Fig. 5). A small portion of the implanted cartilage, approximately $1 \mathrm{~mm}^{2}$ in size and thinner than $0.1 \mathrm{~mm}$, was sampled with the consent of the patient and histological examination revealed a thin synovial layer and matrix with scattered chondrocytes which appeared viable (Fig. 6).

At the most recent review at two years and ten months post-operatively he was pain-free and the range of movement was from $20^{\circ}$ of fixed flexion to $118^{\circ}$ of flexion, $60^{\circ}$

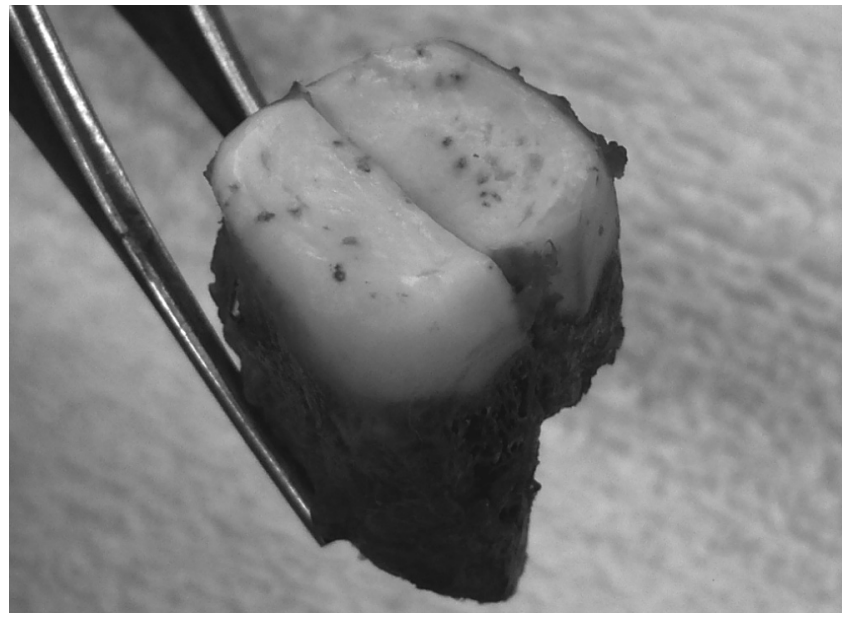

Fig. 3

Photograph showing two pieces of osteochondral graft obtained from the costo-osteochondral junction of the left 4th and 5th rib. They were combined and shaped to form a radial head and neck.

of pronation, and $75^{\circ}$ of supination. The latest radiographs showed satisfactory union between the implanted grafts and the radial neck (Fig. 7). During the postoperative period the patient had undergone a growth spurt resulting in $11 \mathrm{~cm}$ increase in height and $3.5 \mathrm{~cm}$ in the length of the forearm; however no growth disturbance has been observed.

\section{Discussion}

Although fractures of the neck are of the radius are common, post-traumatic osteonecrosis is rare. ${ }^{1}$ Once failure occurs, its management is either conservative or surgical with resection of the radial head at skeletal maturity, but neither option seemed appropriate for our patient, where the radius had already shown signs of proximal migration. 


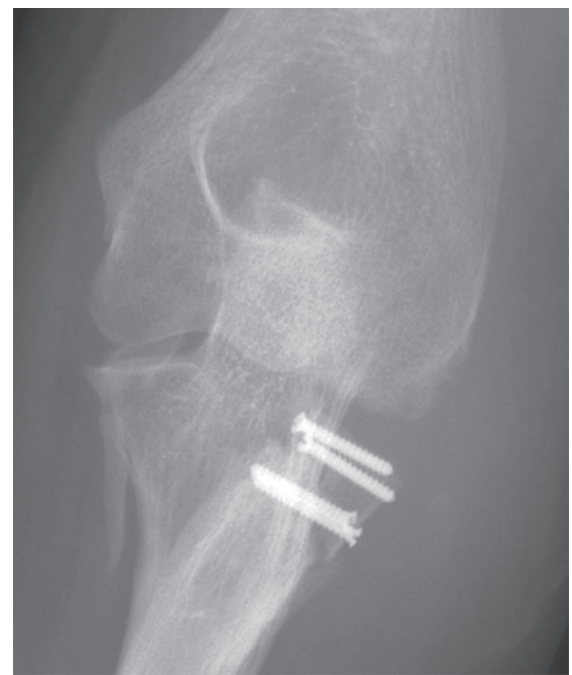

Fig. 4

Post-operative radiograph showing an increased radiolucency of the radial head because of the thickness of the cartilaginous portion of the graft.

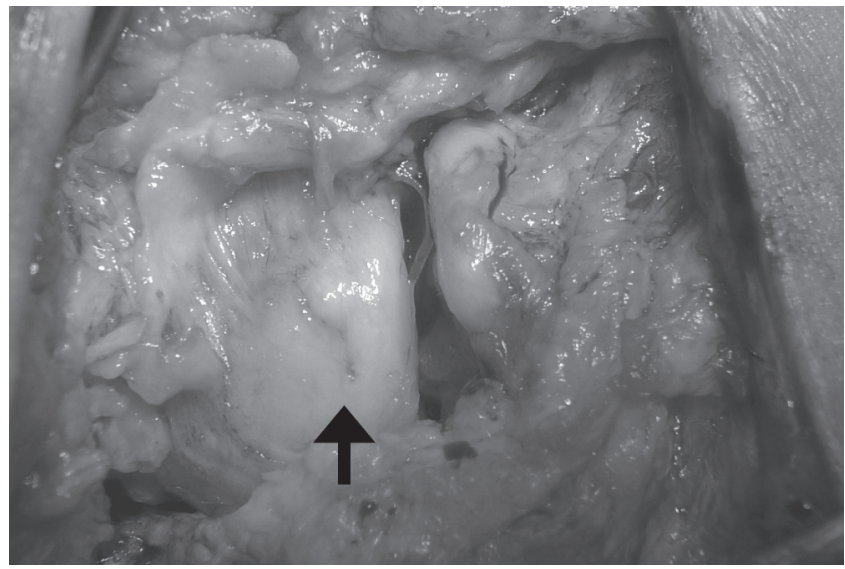

Fig. 5

Intra-operative photograph showing the reconstructed radial head (arrow) seven months after the transplantation. The articular surface is maintained.

Gillies $^{2}$ first described the technique of using costoosteochondral grafts to treat congenital facial deformity by reconstructing the temporomandibular joint. In recent decades, this procedure has been modified to address loss of the proximal pole of the scaphoid, ${ }^{3}$ post-traumatic damage to the articular cartilage in finger joints ${ }^{4}$ and osteochondritis dissecans of the elbow. ${ }^{5}$ We were able to adapt this technique for our patient. Although reconstruction of the radial head using an osteochondral graft has not been previously reported in the English literature, one author (YI) has participated in reporting two cases of comminuted fracture of the radial head treated with costo-osteochondral graft in the Japanese literature. ${ }^{6}$ Kitaoka et $\mathrm{al}^{7}$ noted that costal cartilage shows pheno-

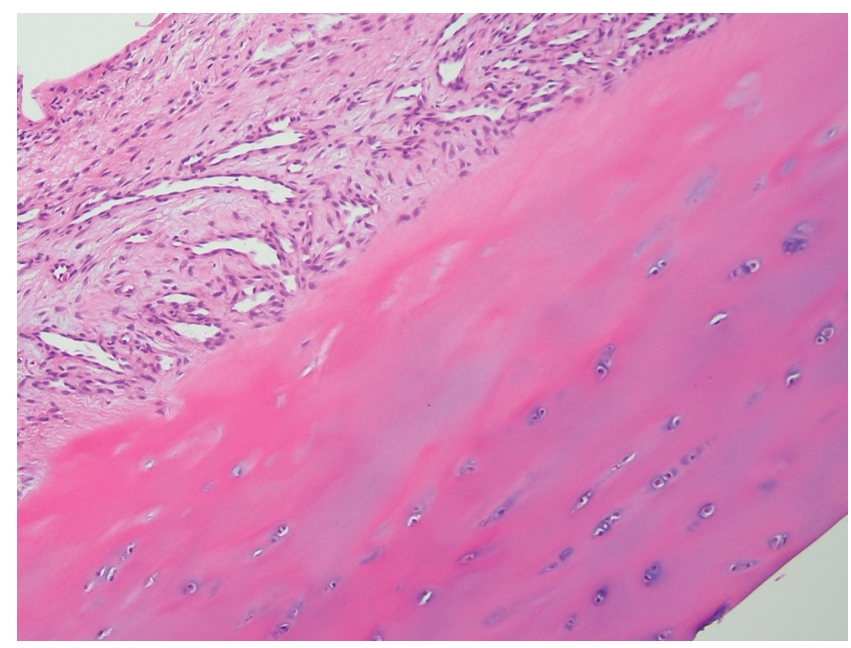

Fig. 6

Histological examination of a sample of the implanted cartilage showing scattered chondrocytes in the matrix resembling normal hyaline cartilage (haematoxylin-eosin stain, magnification $\times 100$ ).

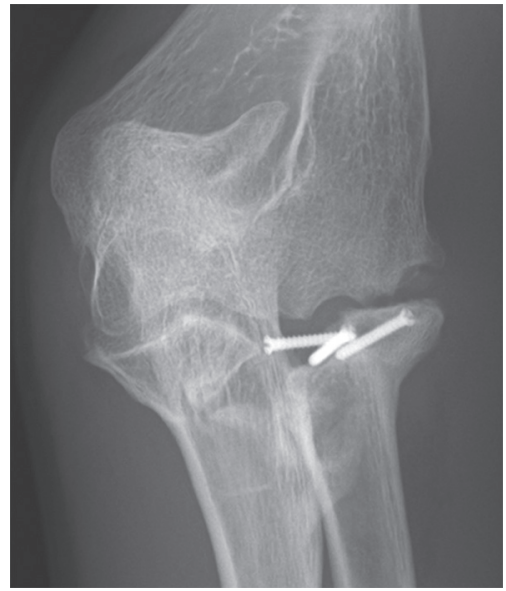

Fig. 7

Radiograph at two years and ten months post-operatively demonstrate bony union of the graft. There has been further erosion of the capitellum.

typic similarities to articular cartilage using chondrocyte cell lines of transgenic mice, suggesting that it might be used on the treatment of articular cartilage defects.

A costo-osteochondral graft allows the defect to be repaired anatomically and biologically with uniform hyaline cartilage. The amount of theharvest depends on the size of the defect to be managed. A part of a junction may be needed to reconstruct the partial defect of a finger joint, while the whole junction may be required to restore defects of the capitellum. Although whole costo-osteochondral junctions may be taken, the donor sites remain uncomfortable for only a few days. It has been reported that the defect at the donor ribs is replaced with hard connective tissue and causes no symptoms, even in athletic activities. ${ }^{4}$ 
Other possible benefits found with our patient include the growth potential of the graft. Although our patient had entered his growth spurt, there was no growth disturbance at the time of the latest review. Longer-term follow-up is needed to confirm the viability and functional outcome of the graft, as well as its growth potential.

No benefits in any form have been received or will be received from a commercial party related directly or indirectly to the subject of this article.

\section{References}

1. Young $\mathbf{S}$, Letts $\mathbf{M}$, Jarvis $\mathbf{J}$. Avascular necrosis of the radial head in children. J Pediatr Orthop 2000;20:15-18.
2. Gillies HD. Plastic surgery of the face. London, Henry Frowde, 1920:177-82.

3. Veitch S, Blake SM, David H. Proximal scaphoid rib graft arthroplasty. J Bone Joint Surg [Br] 2007;89-B:196-201.

4. Sato K, Sasaki T, Nakamura T, Toyama Y, Ikegami H. Clinical outcome and histologic findings of costal osteochondral grafts for cartilage defects in finger joints. $J$ Hand Surg [Am] 2008;33:511-15.

5. Sato K, Nakamura T, Toyama Y, Ikegami H. Costal osteochondral grafts for osteochondritis dissecans of the capitulum humeri. Tech Hand Up Extrem Surg 2008;12:8591.

6. Furushima $\mathbf{K}$, Itoh $\mathbf{Y}$, Tsujino A, et al. Reconstruction of the radial head using a rib with costal cartilage. J Jpn Elbow Soc 2007;14:211-15 (in Japanese).

7. Kitaoka E, Satomura K, Hayashi E, et al. Establishment and characterization of chondrocyte cell lines from the costal cartilage of SV40 large T antigen transgenic mice. J Cell Biochem 2001;81:571-82. 